\title{
Clinical Effects of Xanthine Oxidase Inhibitors in Hyperuricemic Patients
}

\author{
Arrigo F.G. Cicero ${ }^{a, b}$ Federica Fogacci ${ }^{a}$ Raffaele Ivan Cincione ${ }^{c}$ \\ Giuliano Tocci $^{\mathrm{d}, \mathrm{e}}$ Claudio Borghi ${ }^{\mathrm{a}, \mathrm{b}}$
}

${ }^{a}$ Hypertension Research Unit, Medical and Surgical Sciences Department, University of Bologna, Bologna, Italy; bIRCCS Policlinico di S.Orsola, Bologna, Italy; ' Department of Clinical and Experimental Medicine, University of

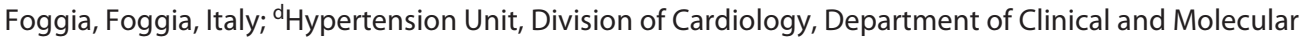
Medicine, Faculty of Medicine and Psychology, University of Rome Sapienza, Sant'Andrea Hospital, Rome, Italy; eIRCCS Neuromed, Pozzilli, Italy

\section{Highlights}

- Hyperuricemia is a well-recognized risk factor for gout and has been shown to contribute to vascular damage.

- Large long-term clinical trials have demonstrated xanthine oxidase inhibitors to be generally effective, safe, and relatively well-tolerated.

- New urate-lowering drugs seem to be particularly efficacious for acute treatment of refractory hyperuricemia, though their use is supported by relatively small clinical evidence.

\section{Keywords}

Uric acid · Hyperuricemia · Uric acid-lowering drugs · Xanthine oxidase inhibitors

\begin{abstract}
This review aims to critically present the available clinical evidence supporting the treatment of chronic hyperuricemia with xanthine oxidase inhibitors. For this reason, the studies published on uric acid (UA)-lowering drugs in the English language from 2000 to August 2019 have been carefully reviewed. The terms "serum uric acid," "xanthine oxidase," "allopurinol," "febuxostat," and "topiroxostat" were incorporated into an electronic search strategy, alone and in combinations, in both MEDLINE (National Library of Medicine, Bethesda, MD) and the Cochrane Register of Controlled Tri-
\end{abstract}

als (The Cochrane Collaboration, Oxford, UK). Even if new urate-lowering drugs seem of particular efficacy for acute treatment of refractory hyperuricemia, their use is supported by relatively small clinical evidence. On the contrary, large long-term clinical trials have demonstrated that xanthine oxidase inhibitors (XOls, namely, allopurinol and febuxostat) are effective, safe, and relatively well-tolerated in most of the patients. They have mainly been tested in the elderly, in patients affected by chronic diseases such as heart failure and cancer, and in patients taking a large number of drugs, confirming their safety profile. Recent data also show that they could exert some positive effects on vascular health, renal function, and glucose metabolism. Their cost is also low. In conclusion, XOls remain the first choice of UA-lowering drug for chronic treatment.

(c) 2020 The Author(s)

Published by S. Karger AG, Basel
(C) 2020 The Author(s)

Published by S. Karger AG, Basel

This is an Open Access article licensed under the Creative Commons Attribution-NonCommercial-4.0 International License (CC BY-NC) (http://www.karger.com/Services/OpenAccessLicense), applicable to the online version of the article only. Usage and distribution for commercial purposes requires written permission.
Arrigo F.G. Cicero

S. Orsola-Malpighi University Hospital

Via Albertoni 15

IT-Bologna (Italy)

arrigo.cicero@unibo.it 


\section{Introduction}

Uric acid (UA) is the end product of exogenous and endogenous pools of purine metabolism. The exogenous pool includes dietetic factors, such as fructose, animal proteins, purine, and alcohol intake, and physiological conditions such as sex, age, renal function, and cell turnover rate [1], whereas the endogenous pool is mainly from the liver, intestines, and other tissues like muscles, kidneys, and the vascular endothelium. UA production and metabolism are processes involving hepatic production as well as renal and gut excretion of this compound. Xanthine oxidase (XO) catalyzes the 2 terminal reactions of purine catabolism in humans. In particular, XO catalyzes the oxidation from hypoxanthine to xanthine and from xanthine to UA, with the simultaneous reduction of $\mathrm{NAD}^{+}$or $\mathrm{O}_{2}$. Therefore, $\mathrm{XO}$ is the housekeeping and the rate-limiting enzyme in purine catabolism. The serum levels of UA (SUA) are kept at a healthy level [2], mainly thanks to the homeostatic regulation involving the renal transport systems. The proximal tubule is the site of reabsorption and secretion of UA, and almost all of it is reabsorbed into the blood. This is primarily accomplished at the proximal tubular level by transporters that exchange intracellular anions for UA. Almost all reabsorption of UA occurs at the $S 1$ segment of the proximal tubule. In the S2 segment of the proximal tubule, UA is secreted to a greater extent than that which undergoes reabsorption. Post-secretory reabsorption occurs at a more distal site of the proximal tubule, and approximately $10 \%$ of the filtered UA appears in the urine. UA is mainly produced in the liver and intestine, being generated from xanthine and hypoxanthine and finally resulting from the purine catabolism. In most living species, such as primates, this metabolic pathway has been highly conserved during the evolutionary process, while some birds and Dalmatian dogs have experienced an increase in circulating levels of UA after losing the functionality of the final step in the degradation of UA [3].

Hyperuricemia may result from the overproduction of urate and mostly from underexcretion of UA, and often from a combination of the both $[4,5]$. Lately, attention has been focused on gut microbiota and ABCG2 expression in the intestine as pathogenic mechanisms [6].

Very high SUA is a well-recognized risk factor for gout, but it should be recognized that SUA levels even below the upper limits of normal seem to increase the chance of developing cardiovascular disease (CVD), type 2 diabetes mellitus, and $\mathrm{CKD}[7,8]$. So, the recognition of a possible independent causal role of hyperuricaemia in

Efficacy and Safety of Xanthine Oxidase Inhibitors the pathogenesis of vascular damage could be of considerable clinical importance, given that the hypouricemic drugs currently available in clinical practice are very effective in reducing levels of circulating UA. The main biological mechanisms by which elevated plasma levels of UA can contribute to vascular damage are the following: prooxidant actions, inducing an increased production of oxygen free radicals and a series of other alterations that are potentially damaging to the vascular wall and other tissues [9]; endothelial dysfunction mediated by the increase in UA through the reduction of endothelial release of nitric oxide and the activation of the renin-angiotensin system with consequent damage at the vascular and renal levels $[10,11]$, endothelial and vascular smooth muscle cells proliferation through the induction of multiple kinases and intracellular growth factors $[12,13]$; increased synthesis and release of endothelin-1; and pro-inflammatory action through activating the transcription of the nuclear factor- $\mathrm{kB}$ to induce the synthesis of tumor necrosis factor- $\alpha$ and interleukin- 1 and expression of various other chemokines including the monocyte chemotactic factor-1 (a chemokine directly involved in the processes of atherogenesis) and through the activation of cyclooxygenase- 2 of protein kinase $C$ and other protein kinases $[14,15]$. Further experimental evidence suggests that hyperuricaemia may also play a causal role in the etiopathogenesis of insulin resistance (a known cardiovascular risk factor) through the induction of systemic endothelial dysfunction and pro-inflammatory and oxidative alterations, especially at the level of adipocytes [16].

Xanthine oxidase inhibitors (XOIs) are UA-lowering drugs with perhaps the most positive effects on cardiovascular outcomes beyond their consolidated positive action on gout risk [11]. This narrative review aims to shortly summarize the pharmacological profile (Table 1) and the main clinical indications (Table 2) of the clinically tested XOIs in hyperuricemic patients.

A recent meta-analysis of 16 randomized clinical trials including 1,211 patients with CKD has shown that SUAlowering therapy halves the relative risk (RR) for kidney failure events; they reduce by more than half the RR for cardiovascular events, though not having a statistically significant impact on the risk of all-cause death [17]. Of note is a recently published case-matched cohort study evaluating the risk of death from CVD and all causes in patients with gout who received or did not receive uratelowering therapy (ULT); this study showed that patients with gout treated with ULT had a lower risk of CVD and all-cause mortality relative to patients with gout not treated with ULT [18]. 
Table 1. Main pharmacological characteristics of the available xanthine oxidase inhibitors

\begin{tabular}{llllll}
\hline Drug & $\begin{array}{l}\text { Oral bioavail- } \\
\text { bility, } \%\end{array}$ & $\begin{array}{l}\text { Protein } \\
\text { binding, } \%\end{array}$ & Metabolism & $\begin{array}{c}\text { Biological half-life, h } \\
\text { Hypouricemic } \\
\text { action, \% }\end{array}$ \\
\hline Allopurinol $\left(\mathrm{C}_{5} \mathrm{H}_{4} \mathrm{~N}_{4} \mathrm{O}\right)$ & $78 \pm 20$ & Negligible & Liver (80\% oxipurinol, 10\% allopurinol ribosides) & $2-3$ (oxipurinol 14-30) & $-24 \pm 2$ \\
\hline Febuxostat $\left(\mathrm{C}_{16} \mathrm{H}_{16} \mathrm{~N}_{2} \mathrm{O}_{3} \mathrm{~S}\right)$ & $>80$ & 99 & $\begin{array}{l}\text { Liver (25-45\% unchanged, 22-44\% febuxostat acyl } \\
\text { glucuronide) }\end{array}$ & $5-8$ & $-27 \pm 3$ \\
\hline Topiroxostat $\left(\mathrm{C}_{13} \mathrm{H}_{8} \mathrm{~N}_{6}\right)$ & 80 & $>92$ & Liver & $4.5-7-5$ & $-44 \pm 5$ \\
\hline
\end{tabular}

Table 2. Main clinical indications of xanthine oxidase inhibitors

\begin{tabular}{|c|c|c|c|c|c|c|}
\hline Allopurinol & Yes & Yes & No & No & Yes & No \\
\hline Febuxostat & Yes & Yes & No & Yes & No & Yes \\
\hline Topiroxostat & Yes & Yes & No & Yes & Yes & Yes \\
\hline
\end{tabular}

\section{Literature Search}

The literature search strategy was based on the availability of clinical pharmacology data and preliminary or advanced clinical data on the efficacy and safety of UAlowering drugs. We searched MEDLINE, Embase, and the Cochrane Database of Systematic Reviews to identify randomized clinical trials, meta-analysis, and systematic reviews published from inception to August 1, 2019 and related to the keywords: "serum uric acid," "xanthine oxidase," "allopurinol," "febuxostat," and "topiroxostat," and only human data were considered and nonhuman experimental data were excluded from the review, as regards the clinical effects of XOIs in hyperuricemic patients. Two authors independently subsequently reviewed all of the citations retrieved from the electronic search in order to identify potentially relevant articles for the present review and determine their eligibility. Quality assessment of each article was performed evaluating the study's aim, case and control definitions, inclusion and exclusion criteria, sample selection and analysis, and statistical definition of significant differential expression. Bibliographies of all identified studies and review articles were reviewed looking for additional papers of interest; no language restriction was used in the literature search. This review represents a careful and updated revision of the most significant literature in the field.

\section{XO Inhibitors}

In the purine metabolism pathway, $\mathrm{XO}$ in the form of xanthine oxidoreductase converts hypoxanthine into UA. This process brings about the production of reactive oxygen species, which in excess reduces nitric oxide synthesis, finally leading to endothelial dysfunction. A recent meta-analysis of 81 randomized clinical trials overall involving 10,684 patients showed that XOIs significantly reduce the risk of total and serious cardiovascular events $(\mathrm{OR}=0.60,95 \% \mathrm{CI}[0.44,0.82]$ and $\mathrm{OR}=0.64,95 \% \mathrm{CI}$ $[0.46,0.89]$, respectively) and onset hypertension $(\mathrm{OR}=$ $0.54,95 \%$ CI $[0.37,0.80])$ when compared to placebo. Furthermore, a sub-analysis carried out on 9 studies and 616 hyperuricemic subjects showed that XOIs are more effective in secondary prevention, significantly reducing the occurrence of major adverse cardiovascular events in individuals with previous transient ischemic attacks, stroke, unstable angina, or myocardial infarction $(\mathrm{RR}=$ $0.42,95 \%$ CI $[0.23,0.76] ; p<0.01 ; I^{2}=0 \%$ ) [19]. These potential benefits attributed to the XOIs may rely on their antioxidant properties other than SUA reduction as they inhibit the production of reactive oxygen species by the $\mathrm{XO}[20]$. Also, these agents are the first-line drugs in ULT for gout, being effective in overproducers as well as in under-excretors of UA [21]. 


\section{Allopurinol}

Allopurinol and its metabolite oxypurinol are analogs of hypoxanthine and xanthine, respectively, and prevent the formation of UA by binding to XO and inhibiting it [22]. Allopurinol is an orally and parenterally administrable drug for the treatment of gout and preventing the recurrence of kidney stones. Treatment with allopurinol has been associated with an improvement in the flowmediated dilation [23] and a slowdown in the progression of CKD [24, 25]. Moreover, it is the mainstay of prophylactic treatment for hyperuricemia in patients undergoing chemotherapy [26].

Immediately after oral administration, allopurinol is quickly absorbed in the upper digestive tract. It reaches peak plasma concentrations in about $30 \mathrm{~min}$ after ingestion and has a plasma half-life of about $2-3 \mathrm{~h}$. The primary active metabolite of allopurinol is oxypurinol, which is filtered and partially reabsorbed in the kidneys. Oxypurinol has the same mechanism of action of allopurinol, but a longer plasma half-life of $14-30 \mathrm{~h}$ and lower oral bioavailability than its precursor [27].

Allopurinol has a dose-dependent effect of lowering serum urate levels, and the usual daily dosages given for the treatment of chronic hyperuricemia range from 100 to $600 \mathrm{mg}$ [28]. The maximal daily allopurinol dose may, however, be as much as $800-900 \mathrm{mg}$ based on the country and product label [29]. A well-designed meta-analysis has shown that only lower doses of allopurinol $(\leq 300 \mathrm{mg} /$ day) can reduce the risk of total cardiovascular events, while subjects treated with allopurinol at higher doses do not seem to have any significant reduction in risk [11]. The explanation for this could be that higher doses of allopurinol may lead to loss of cardiovascular protection, considering that higher levels of oxypurinol may promote oxidative stress, as suggested by the study of Stamp et al. [30]. They observed that higher concentrations of oxypurinol promote a switch from an antioxidant to a prooxidant state, given that oxypurinol is a suitable substrate for myeloperoxidase (released by neutrophils in inflammatory states such as gout and hyperuricemia), generating radicals capable of oxidizing urate and promoting deleterious effects.

In an observational study carried out on 6,428 dose escalators and as many non-escalators, dose escalation has been associated with a small increase $(<10 \%)$ in all-cause mortality, showing that it is unlikely to improve 10 -year survival [31]. However, it is generally recommended that patients start with a low dose of allopurinol and increase it gradually. This conservative approach significantly lowers the risk of potentially fatal hypersensitivity syndrome

Efficacy and Safety of Xanthine Oxidase Inhibitors and helps to prevent acute gout attacks, which tend to occur immediately after starting the treatment; gradual titration of the dosage has been shown to mitigate this effect [32]. However, in order to decrease the risk of acute attacks of gout, the administration of anti-inflammatory drugs or low-dose colchicine can be useful as needed during the initiation of therapy with allopurinol [33].

The most common documented side effects are gastrointestinal distress and skin rash that can range in severity. Apart from potentially severe skin reactions, serious side effects include allopurinol hypersensitivity syndrome, a rare as well as potentially fatal condition consisting of eosinophilia, hepatitis, and interstitial nephritis. In particular, subjects starting on high doses of allopurinol and CKD patients in treatment with thiazide diuretics are at increased risk for developing allopurinol hypersensitivity syndrome; clinicians therefore tend to use lower ranges for maximum dosing in patients with renal impairment. Moreover, pharmacogenomics plays a role in the side effect profile of allopurinol, and the risk of severe side effects increases in patients with the HLAB*5801 haplotype, commonly found in Han Chinese and Thai ethnicities other than in Korean descents with at least stage 3 CKD [34]. Some of these concerns can lead to allopurinol underdosing, consequently resulting in ineffective control of hyperuricemia [35].

Finally, allopurinol is contraindicated in patients on didanosine [36]. Also, the concomitant use of allopurinol at a dose of $300-600 \mathrm{mg} /$ day with mercaptopurine or azathioprine requires a reduction in dose of these agents to approximately one-third or one-fourth of their usual dose. Therapeutic response and toxicities should be monitored accordingly [37].

\section{Febuxostat}

Febuxostat is an oral non-purine selective XO inhibitor that can inhibit both the oxidized and the reduced form of XO by binding them. Unlike allo/oxypurinol, febuxostat prevents enzyme turnover, avoiding the consequent production of ROS by blocking the active pterinmolybdenum center of the enzyme-substrate complex [38]. After oral administration, it is absorbed in the upper digestive tract and achieves its peak plasma concentrations within an hour, having a plasma half-life of around 5-8 h [30]. Febuxostat is metabolized and mainly excreted through the hepatic conjugation processes. In the past, several studies have clarified that it is more powerful and effective than allopurinol in lowering SUA levels and exerting anti-inflammatory effects on the endothelial cells $[39,40]$. Its efficacy has been partly related to its ability to 
inhibit glycosaminoglycan-bound and endothelial cellbound XO [41]. However, febuxostat seems to exert a different inhibitory effect on $\mathrm{XO}$ in terms of central and peripheral endothelial function. A study comparing the effect of febuxostat and allopurinol in patients with gout has shown that febuxostat is more effective in preventing peripheral arterial stiffening as measured by carotid-femoral pulse wave velocity over 1 year [42]. On the contrary, a recent phase 4 randomized, placebo-controlled, doubleblind, crossover trial has found that febuxostat does not significantly improve coronary endothelial dysfunction in patients with known stable cardiovascular artery disease and magnetic resonance evidence, though it lowers SUA levels [43].

The recommended dosage of febuxostat is $80-120 \mathrm{mg} /$ day, even though remarkable decreases in SUA levels have also been observed at the lower dose of $40 \mathrm{mg} /$ day. In particular, the number of subjects who can reach target SUA values $(<6 \mathrm{mg} / \mathrm{dL})$ with $40 \mathrm{mg}$ febuxostat seems to be more than the number of subjects in treatment with allopurinol $300 \mathrm{mg}$ [44]. Febuxostat is indicated for the treatment of hyperuricemia in gout patients, being a valid alternative therapy for individuals in whom allopurinol is contraindicated due to a previous allergic reaction [45]. On the contrary, it is not indicated in asymptomatic hyperuricemic patients since evidence is lacking [46].

Recent findings have shown that no dosage adjustments are necessary in patients with mild-to-moderate or severe hepatic and renal impairment (with $\mathrm{CrCl}<30 \mathrm{~mL}$ / min), even if this drug has not just been studied in patients on dialysis. In general, side effects associated with febuxostat include muscle pain, stomach pain, diarrhea, and a slight elevation in liver enzymes [47]. A meta-analysis of available comparative clinical trials showed that febuxostat tolerability is overall significantly higher than that of allopurinol in patients with hyperuricemia or gout [48] and especially in the ones with renal failure, having a lower incidence of adverse events (OR 0.85, 95\% CI $[0.75,0.97])$ [49]. Febuxostat is also supposed to have nephroprotective activity compared to allopurinol [50] and has recently been demonstrated to effectively and safely reduce SUA levels also in kidney transplant patients [51, 52]. For these reasons, even if 2016 updated European League Against Rheumatism (EULAR) guidelines suggest the use of febuxostat as second-line SUA-lowering agent for the patient either not responding or intolerant to allopurinol [53], the latest guidelines - such as the guidelines of the American College of Physicians (ACP 2017) - consider febuxostat as a first-line agent in hyperuricemia treatment [54].
According to a lately published meta-analysis carried out on 10 trials, including 14,402 patients, febuxostat is neutral toward cardiovascular events [55]. However, based on the largely criticized results of the more recent "Cardiovascular Safety of Febuxostat and Allopurinol in Patients with Gout and Cardiovascular Morbidities (CARES)" trial [56], the Food and Drug Administration to limit the approved use of febuxostat to patients who are not treated effectively or experience severe side effects with allopurinol, suggesting special attention to patients at high risk for cardiovascular events, already assuming the drug [57]. Besides, in the recent "Febuxostat for Cerebral and CaRdiorenovascular Events PrEvEntion StuDy - FREED," no negative impact of febuxostat on cardiovascular events has been observed [58], while in a recent observational study carried out on mild-to-moderate heart failure patients, febuxostat seems to have a positive impact on cardiovascular mortality compared with allopurinol [59]. Finally, a recent meta-analysis of randomized clinical trials showed no difference between allopurinol and febuxostat in terms of association with major cardiovascular events [60].

\section{Topiroxostat}

Approved for therapeutic use in Japan since 2013, topiroxostat is another selective XOI with good oral bioavailability in humans. Its pharmacologically active metabolite (1,000 times less than the parent drug) is the $\mathrm{N}$ glucuronidated topiroxostat (F11741), which is produced in the liver [61]. In $\mathrm{db} / \mathrm{db}$ mice, this drug has been shown to cause a dose-dependent reduction in both urinary albumin excretion and plasma XO activity [62]. Similar findings have been safely confirmed in hyperuricemic patients with CKD stage III, in whom topiroxostat $160 \mathrm{mg}$ was reported to effectively decrease SUA and urinary albumin excretion [63].

The "Effects of Topiroxostat for hyperUricaemic patients with overt Diabetic nEphropathy (ETUDE)" study [64] and the "Uric acid-lowering and renoProtective effects of topiroxostat in patients With diAbetic nephropAthy and hyperuRicemia (UPWARD)" study [65] have confirmed the positive impact of topiroxostat on renal function in patients with overt diabetic nephropathy. Overall, these results suggest topiroxostat may have a renal protective effect over and above its UA-lowering action [66].

The long-term (58 weeks) efficacy and tolerability have been further confirmed in a hyperuricemic patient with or without gout [67]. The results of a new trial (Effect of Xanthine Oxidase Inhibitor in Chronic Heart Fail- 
ure Patients Complicated with Hyperuricemia study Excited-UA) study carried out on heart failure patients are soon expected [68]. Topiroxostat has also been demonstrated to significantly and safely decrease SUA levels in hyperuricemic patients receiving hemodialysis at the minimum dose compared to allopurinol ones [69]. Moreover, since topiroxostat is not dialyzable, unlike the other $\mathrm{XOIs}$, the dose reduction is not required even in patients with lowered renal function [70]. While the preliminary clinical data are promising, larger long-term studies are required to better characterize the efficacy and safety profile of topiroxostat [71].

\section{3,4-Dihydroxy-5-Nitrobenzaldehyde}

3,4-Dihydroxy-5-nitrobenzaldehyde (DHNB) is a new and powerful time-dependent XOI with a mechanism of action still under investigation, but that seems to be similar to allopurinol. Some studies have suggested that DHNB has low toxicity and enhances therapeutic efficacy, even at low doses, in co-administration with allopurinol. Moreover, DHNB has a direct antioxidant capacity and reduces the production of free radicals and ROS at source, in order to limit cell damage. However, while it appears to be extremely safe and effective in mice, no human data are available yet [72].

\section{Concluding Remarks}

A clear role for SUA level has been shown convincingly only for gout and nephrolithiasis, but the possible role of hyperuricaemia in the pathogenesis of CVD continues to be a subject of intense scientific debate, as it is not completely clear whether the increase in uremia is simply a marker or a true cardiovascular risk factor $[1,5$, 6]. However, hyperuricemia also seems to be an independent risk factor for developing type 2 diabetes, CKD, hypertension, atrial fibrillation, coronary artery disease, and heart failure; it also predisposes to gout, so optimizing SUA levels is increasing as a public health priority [73, 74]. Currently, there is no general agreement on lifestyle modification, which might be more effective in reducing SUA levels, insofar as a low-energy Mediterranean diet aimed at achieving optimal body weight seems reasonably the best approach [75].

The available urate-lowering drugs can be grouped by their mechanism of SUA reduction; XOIs reduce urate production, while the uricosuric agents probenecid, benzbromarone, sulfinpyrazone, and lesinurad increase renal excretion of SUA by inhibiting its reabsorption. Fi- nally, the injectable uricase enzymatically degrades UA to allantoin [76].

The XOIs are undoubtedly the most studied both for their ability to reduce SUA and for their extra-UA effects [77]. A meta-analysis of 24 studies has shown that adherence to ULT among gout patients is poor, suggesting the need for more attention by clinicians [78]. Furthermore, considering the amount of cardiovascular risk associated with hyperuricemia, the use of lipid-lowering and antihypertensive drugs (such as fenofibrate and losartan), which are able to improve SUA levels, is recommendable, insofar as the use of these drugs has not just been clearly demonstrated to reduce the SUA-related risk for human health beyond their effects on lipids and blood pressure [13]. Certainly, this approach should be even more rigorous in diabetic patients because the use of SGLT2 inhibitors rather than other antidiabetic drugs has been shown to reduce SUA by up to $-0.63 \mathrm{mg} / \mathrm{dL}$ ( $95 \%$ CI $[-0.98$, $-0.59]$ ] [79]. At the same time, medications increasing SUA levels, such as old-generation $\beta$-blockers [80] and high-dose thiazides [81], should be substituted with other metabolically neutral antihypertensive drugs. Finally, considering the need for chronic treatment, the choice of an SUA-lowering drug should be oriented both by the knowledge of the pathophysiological mechanism of the disease and by consideration about product safety and tolerability [82], other than its positive effect on the cardiometabolic and renal outcome measures [83].

Even if new urate-lowering drugs seem of particular efficacy for acute treatment of refractory hyperuricemia, their use is supported by relatively small clinical evidence [84]. On the contrary, large long-term clinical trials have clearly demonstrated that XOIs (namely, allopurinol and febuxostat) are effective, safe, and relatively well-tolerated in the most patients [55]. They have been largely tested in the elderly, in patients affected by chronic diseases such as heart failure and cancer, and in patients taking a large number of drugs, confirming their safety profile. In conclusion, XO remains the first choice of UA-lowering drug for chronic treatment.

\section{Conflict of Interest Statement}

C.B. served as a consultant for Menarini International. The other authors declare no conflicts of interest.

\section{Funding Sources}

The authors did not receive any funding. 


\section{References}

1 Katsiki N, Karagiannis A, Athyros VG, Mikhailidis DP. Hyperuricaemia: more than just a cause of gout? J Cardiovasc Med. 2013; 14(6):397-402.

2 Jin M, Yang F, Yang I, Yin Y, Luo JJ, Wang H, et al. Uric acid, hyperuricemia and vascular diseases. Front Biosci. 2012;17:656-69.

3 Oda M, Satta Y, Takenaka O, Takahata N. Loss of urate oxidase activity in hominoids and its evolutionary implications. Mol Biol Evol. 2002;19(5):640-53.

4 Keenan RT. Limitations of the current standards of care for treating gout and crystal deposition in the primary care setting: a review. Clin Ther. 2017;39(2):430-41.

5 Cicero AF, Rosticci M, Fogacci F, Grandi E, D'Addato S, Borghi C. Brisighella Heart Study Group: high serum uric acid is associated to poorly controlled blood pressure and higher arterial stiffness in hypertensive subjects. Eur J Intern Med. 2017;37:38-42.

6 Pascart T, Lioté F. Gout: state of the art after a decade of developments. Rheumatology. 2018;58(1):27-44.

7 Galassi FM, Borghi C. A brief history of uric acid: from gout to cardiovascular risk factor. Eur J Intern Med. 2015;26(5):373.

8 Bos MJ, Koudstaal PJ, Hofman A, Witteman JC, Breteler MM. Uric acid is a risk factor for myocardial infarction and stroke: the Rotterdam study. Stroke. 2006;37(6):1503-7.

9 Lippi G, Montagnana M, Franchini M, Favaloro EJ, Targher G. The paradoxical relationship between serum uric acid and cardiovascular disease. Clin Chim Acta. 2008;392(1-2): $1-7$.

10 Zharikov S, Krotova K, Hu H, Baylis C, Johnson RJ, Block ER, et al. Uric acid decreases NO production and increases arginase activity in cultured pulmonary artery endothelial cells. Am J Physiol Cell Physiol. 2008;295(5): C1183-90.

11 Mercuro G, Vitale C, Cerquetani E, Zoncu S, Deidda M, Fini M, et al. Effect of hyperuricemia upon endothelial function in patients at increased cardiovascular risk. Am J Cardiol. 2004;94(7):932-5.

12 Kang DH, Han L, Ouyang X, Kahn AM, Kanellis J, Li P, et al. Uric acid causes vascular smooth muscle cell proliferation by entering cells via a functional urate transporter. Am J Nephrol. 2005;25(5):425-33.

13 Corry DB, Eslami P, Yamamoto K, Nyby MD, Makino H, Tuck ML. Uric acid stimulates vascular smooth muscle cell proliferation and oxidative stress via the vascular renin-angiotensin system. J Hypertens. 2008;26(2):269-75.

14 Han HJ, Lim MJ, Lee YJ, Lee JH, Yang IS, Taub M. Uric acid inhibits renal proximal tubule cell proliferation via at least two signaling pathways involving PKC, MAPK, cPLA2, and NF-kappaB. Am J Physiol Renal Physiol. 2007;292(1):F373-81.
15 Kanellis J, Watanabe S, Li JH, Kang DH, Li P, Nakagawa T, et al. Uric acid stimulates monocyte chemoattractant protein-1 production in vascular smooth muscle cells via mitogen-activated protein kinase and cyclooxygenase- 2 . Hypertension. 2003;41(6):1287-93.

16 Sautin YY, Nakagawa T, Zharikov S, Johnson RJ. Adverse effects of the classic antioxidant uric acid in adipocytes: NADPH oxidase-mediated oxidative/nitrosative stress. Am J Physiol Cell Physiol. 2007;293(2):C584-96.

17 Su X, Xu B, Yan B, Qiao X, Wang L. Effects of uric acid-lowering therapy in patients with chronic kidney disease: a meta-analysis. PLoS One. 2017;12(11):e0187550.

18 Chen JH, Lan JL, Cheng CF, Liang WM, Lin HY, Tsay GJ, et al. Effect of urate-lowering therapy on the risk of cardiovascular disease and all-cause mortality in patients with gout: a case-matched cohort study. J Rheumatol. 2015;42(9): 1694

19 Bredemeier M, Lopes LM, Eisenreich MA, Hickmann S, Bongiorno GK, d'Avila R, et al. Xanthine oxidase inhibitors for prevention of cardiovascular events: a systematic review and meta-analysis of randomized controlled trials. BMC Cardiovasc Disord. 2018;18(1): 24.

20 Okafor ON, Farrington K, Gorog DA. Allopurinol as a therapeutic option in cardiovascular disease. Pharmacol Ther. 2017;172:139_ 50.

21 Bove M, Cicero AF, Veronesi M, Borghi C. An evidence-based review on urate-lowering treatments: implications for optimal treatment of chronic hyperuricemia. Vasc Health Risk Manag. 2017;13:23-8.

22 Day RO, Graham GG, Hicks M, McLachlan AJ, Stocker SL, Williams KM. Clinical pharmacokinetics and pharmacodynamics of allopurinol and oxypurinol. Clin Pharmacokinet. 2007;46(8):623-44.

23 Cicero AFG, Pirro M, Watts GF, Mikhailidis DP, Banach M, Sahebkar A. Effects of allopurinol on endothelial function: a systematic review and meta-analysis of randomized placebo-controlled trials. Drugs. 2018;78(1):99109.

24 Siu YP, Leung KT, Tong MK, Kwan TH. Use of allopurinol in slowing the progression of renal disease through its ability to lower serum uric acid level. Am J Kidney Dis. 2006; 47(1):51-9.

25 Goicoechea M, de Vinuesa SG, Verdalles U, Ruiz-Caro C, Ampuero J, Rincón A, et al. Effect of allopurinol in chronic kidney disease progression and cardiovascular risk. Clin J Am Soc Nephrol. 2010;5(8):1388-93.

26 Allopurinol tablet [Accord Healthcare Inc. available on the Daily Medwebsite. Accessed 2018 Oct 9. Available from: http://dailymed. nlm.nih.gov/dailymed/lookup.cfm?setid = 682dd8b8-fc6e-47c5--95b7--2d7ad96b750.
27 Pea F. Pharmacology of drugs for hyperuricemia: mechanisms, kinetics and interactions. Contrib Nephrol. 2005;147:35-46.

28 Day RO, Graham GG, Hicks M, McLachlan AJ, Stocker SL, Williams KM. Clinical pharmacokinetics and pharmacodynamics of allopurinol and oxypurinol. Clin Pharmacokinet. 2007;46(8):623-44

29 Chao J, Terkeltaub R. A critical reappraisal of allopurinol dosing, safety, and efficacy for hyperuricemia in gout. Curr Rheumatol Rep. 2009;11(2):135-40.

30 Stamp LK, Turner R, Khalilova IS, Zhang M, Drake J, Forbes LV, et al. Myeloperoxidase and oxidation of uric acid in gout: implications for the clinical consequences of hyperuricaemia. Rheumatology. 2014;53(11): 1958-65.

31 Coburn BW, Michaud K, Bergman DA, Mikuls TR. Allopurinol dose escalation and mortality among patients with gout: a national propensity-matched cohort study. Arthritis Rheumatol. 2018;70(8):1298-307.

32 Stamp LK, Taylor WJ, Jones PB, Dockerty JL, Drake J, Frampton C, et al. Starting dose is a risk factor for allopurinol hypersensitivity syndrome: a proposed safe starting dose of allopurinol. Arthritis Rheum. 2012;64(8): 2529-36

33 Chung Y, Stocker SL, Graham GG, Day RO. Optimizing therapy with allopurinol: factors limiting hypouricemic efficacy. Am J Med Sci. 2008;335(3):219-26.

34 Hung SI, Chung WH, Liou LB, Chu CC, Lin M, Huang HP, et al. HLA-B*5801 allele as a genetic marker for severe cutaneous adverse reactions caused by allopurinol. Proc Natl Acad Sci U S A. 2005;102(11):4134-9.

35 Stamp LK, O'Donnell JL, Zhang M, James J, Frampton C, Barclay ML, et al. Using allopurinol above the dose based on creatinine clearance is effective and safe in patients with chronic gout, including those with renal impairment. Arthritis Rheum. 2011;63(2):41221.

36 Panel on Antiretroviral Guidelines for Adults and Adolescents. Guidelines for the use of antiretroviral agents in HIV-1-infected adults and adolescents. Department of Health and Human Services. Last accessed 2019 Sep 1. Available from: http://www.aidsinfo.nih.gov/ ContentFiles/AdultandAdolescentGL.pdf.

37 Allopurinol oral tablets [package insert]. Memphis, TN: Northstar Rx LLC. 2015.

38 Pascual E, Sivera F, Yasothan U, Kirkpatrick P. Febuxostat. Nat Rev Drug Discov. 2009; 8(3):191-2.

39 Becker MA, Schumacher HR Jr, Wortmann RL, MacDonald PA, Eustace D, Palo WA, et al. Febuxostat compared with allopurinol in patients with hyperuricemia and gout. N Engl J Med. 2005;353(23):2450-61. 
40 Schumacher HR Jr, Becker MA, Wortmann RL, Macdonald PA, Hunt B, Streit J, et al. Effects of febuxostat versus allopurinol and placebo in reducing serum urate in subjects with hyperuricemia and gout: a 28-week, phase III, randomized, double-blind, parallel-group trial. Arthritis Rheum. 2008;59(11):1540-8.

41 Malik UZ, Hundley NJ, Romero G, Radi R, Freeman BA, Tarpey MM, et al. Febuxostat inhibition of endothelial-bound XO: implications for targeting vascular ROS production. Free Radic Biol Med. 2011;51(1):179-84.

42 Tausche AK, Christoph M, Forkmann M, Richter U, Kopprasch S, Bielitz C, et al. As compared to allopurinol, urate-lowering therapy with febuxostat has superior effects on oxidative stress and pulse wave velocity in patients with severe chronic tophaceous gout. Rheumatol Int. 2014;34(1):101-9.

43 Hays AG, Iantorno M, Schär M, Lai S, Czarny $\mathrm{M}$, Breton E, et al. The influence of febuxostat on coronary artery endothelial dysfunction in patients with coronary artery disease: a phase 4 randomized, placebo-controlled, doubleblind, crossover trial. Am Heart J. 2018;197: 85-93.

44 Singh JA, Akhras KS, Shiozawa A. Comparative effectiveness of urate lowering with febuxostat versus allopurinol in gout: analyses from large U.S. managed care cohort. Arthritis Res Ther. 2015;17:120.

45 Uloric (febuxostat) drug label available on the Daily Med website. Last accessed 2010 Aug 1. Available from: http://dailymed.nlm.nih.gov/ dailymed/lookup.cfm?setid $=54$ de10ef-fe5f4930-b91d-6bbb04c664bd.

46 Stamp L, Dalbeth N. Urate-lowering therapy for asymptomatic hyperuricaemia: a need for caution. Semin Arthritis Rheum. 2017;46(4): $457-64$.

47 Uloric [package insert]. Deerfield, IL: Takeda Pharmaceuticals America, Inc. 2013.

48 Liu CW, Chang WC, Lee CC, Shau WY, Hsu FS, Wang ML, et al. The net clinical benefits of febuxostat versus allopurinol in patients with gout or asymptomatic hyperuricemia: a systematic review and meta-analysis. Nutr Metab Cardiovasc Dis. 2019;29(10):1011-22.

49 Borghi C, Perez-Ruiz F. Urate lowering therapies in the treatment of gout: a systematic review and meta-analysis. Eur Rev Med Pharmacol Sci. 2016;20(5):983-92.

50 Lin TC, Hung LY, Chen YC, Lo WC, Lin CH, Tam KW, et al. Effects of febuxostat on renal function in patients with chronic kidney disease: a systematic review and meta-analysis. Medicine. 2019;98(29):e16311.

51 Kim S, Kim HJ, Ahn HS, Oh SW, Han KH, $\mathrm{Um} \mathrm{TH}$, et al. Renoprotective effects of febuxostat compared with allopurinol in patients with hyperuricemia: a systematic review and meta-analysis. Kidney Res Clin Pract. 2017; 36(3):274-81.

52 Baek CH, Kim H, Yang WS, Han DJ, Park SK. Efficacy and safety of febuxostat in kidney transplant patients. Exp Clin Transplant. 2018;16(4):401-6.
53 Richette P, Doherty M, Pascual E, Barskova V, Becce F, Castañeda-Sanabria J, et al. 2016 updated EULAR evidence-based recommendations for the management of gout. Ann Rheum Dis. 2017;76(1):29-42.

54 Qaseem A, Harris RP, Forciea MA. For the clinical guidelines committee of the American College of Physicians. Management of acute and recurrent gout: a clinical practice guideline from the American College of Physicians. Ann Internal Med. 2017;166:58-68.

55 Cuenca JA, Balda J, Palacio A, Young L, Pillinger MH, Tamariz L. Febuxostat and cardiovascular events: a systematic review and meta-analysis. Int J Rheumatol. 2019;2019: 1076189.

56 White WB, Saag KG, Becker MA, Borer JS, Gorelick PB, Whelton A, et al. CARES investigators: cardiovascular safety of febuxostat or allopurinol in patients with gout. $\mathrm{N}$ Engl J Med. 2018;378(13):1200-10.

57 Last accessed 2010 Aug 1. Available from: https: //www.fda.gov/drugs/drug-safety-andavailability/fda-adds-boxed-warning-increased-risk-death-gout-medicine-uloric-febuxostat.

58 Kojima S, Matsui K, Hiramitsu S, Hisatome I, Waki M, Uchiyama K, et al. Febuxostat for Cerebral and CaRdiorenovascular Events PrEvEntion StuDy. Eur Heart J. 2019;40(22): 1778-86.

59 Cicero AFG, Cosentino ER, Kuwabara M, Degli Esposti D, Borghi C. Effects of allopurinol and febuxostat on cardiovascular mortality in elderly heart failure patients. Intern Emerg Med. 2019;14(6):949-56

60 Barrientos-Regala M, Macabeo RA, RamirezRagasa R, Pestaño NS, Punzalan FER, Tumanan-Mendoza B, et al. The association of febuxostat compared to allopurinol on blood pressure and major adverse cardiac events (MACE) among adult patients with hyperuricemia: a meta-analysis. J Cardiovasc Pharmacol. 2020 Oct;76(4):461-71.

61 Hosoya T, Sasaki T, Ohashi T. Clinical efficacy and safety of topiroxostat in Japanese hyperuricemic patients with or without gout: a randomized, double-blinded, controlled phase 2b study. Clin Rheumatol. 2017;36(3): 649-56.

62 Nakamura T, Murase T, Nampei M, Morimoto N, Ashizawa N, Iwanaga T, et al. Effects of topiroxostat and febuxostat on urinary albumin excretion and plasma xanthine oxidoreductase activity in $\mathrm{db} / \mathrm{db}$ mice. Eur J Pharmacol. 2016;780:224-31.

63 Hosoya T, Ohno I, Nomura S, Hisatome I, Uchida S, Fujimori S, et al. Effects of topiroxostat on the serum urate levels and urinary albumin excretion in hyperuricemic stage 3 chronic kidney disease patients with or without gout. Clin Exp Nephrol. 2014;18(6):87684.
64 Mizukoshi T, Kato S, Ando M, Sobajima H, Ohashi N, Naruse T, et al. Renoprotective effects of topiroxostat for hyperuremic patients with overt diabetic nephropathy study (ETUDE Study): a prospective, randomized, multicenter clinical trial. Nephrology. 2018; 23(11):1023-30.

65 Wada T, Hosoya T, Honda D, Sakamoto R, Narita K, Sasaki T, et al. Uric acid-lowering and renoprotective effects of topiroxostat, a selective xanthine oxidoreductase inhibitor, in patients with diabetic nephropathy and hyperuricemia: a randomized, double-blind, placebo-controlled, parallel-group study (UPWARD study). Clin Exp Nephrol. 2018; 22(4):860-70.

66 Katsuyama H, Yanai H, Hakoshima M. Renoprotective effect of xanthine oxidase inhibitor, topiroxostat. J Clin Med Res. 2019;11(8): 614-6.

67 Hosoya T, Ishikawa T, Ogawa Y, Sakamoto R, Ohashi T. Multicenter, open-label study of long-term topiroxostat (FYX-051) administration in Japanese hyperuricemic patients with or without gout. Clin Drug Investig. 2018;38(12):1135-43.

68 Sakuma M, Toyoda S, Arikawa T, Koyabu Y, Kato T, Adachi T, et al. For Excited UA study investigators: the effects of xanthine oxidase inhibitor in patients with chronic heart failure complicated with hyperuricemia: a prospective randomized controlled clinical trial of topiroxostat vs allopurinol-study protocol. Clin Exp Nephrol. 2018;22(6):1379-86.

69 Nagaoka Y, Tanaka Y, Yoshimoto H, Suzuki $\mathrm{R}$, Ryu K, Ueda M, et al. The effect of small dose of topiroxostat on serum uric acid in patients receiving hemodialysis. Hemodial Int. 2018;22(3):388-93.

70 Greig D, Alcaino H, Castro PF, Garcia L, Verdejo HE, Navarro M, et al. Xanthine-oxidase inhibitors and statins in chronic heart failure: effects on vascular and functional parameters. J Heart Lung Transplant. 2011; 30(4):408-13.

71 Strilchuk L, Fogacci F, Cicero AF. Safety and tolerability of available urate-lowering drugs: a critical review. Expert Opin Drug Saf. 2019; 18(4):261-71.

72 Lü JM, Yao Q, Chen C. 3,4-Dihydroxy-5-nitrobenzaldehyde (DHNB) is a potent inhibitor of xanthine oxidase: a potential therapeutic agent for treatment of hyperuricemia and gout. Biochem Pharmacol. 2013;86(9):132837.

73 Borghi C, Cicero AFG. Serum uric acid and acute coronary syndrome: is there a role for functional markers of residual cardiovascular risk? Int J Cardiol. 2018 Jan 1;250:62-3.

74 Borghi C, Cicero AFG. Uric acid and early prevention of vascular diseases: women under the looking glass. Int J Cardiol. 2018;272:3145.

75 Beyl RN Jr, Hughes L, Morgan S. Update on importance of diet in gout. Am J Med. 2016 Nov;129(11):1153-8. 
76 Fleischmann R, Winkle P, Miner JN, Yan X, Hicks L, Valdez S, et al. Pharmacodynamic and pharmacokinetic effects and safety of verinurad in combination with allopurinol in adults with gout: a phase IIa, open-label study. RMD Open. 2018;4(1):e000584.

77 Robinson PC. Gout: an update of aetiology, genetics, co-morbidities and management. Maturitas. 2018;118:67-73.

78 Scheepers LEJM, van Onna M, Stehouwer CDA, Singh JA, Arts ICW, Boonen A. Medication adherence among patients with gout: a systematic review and meta-analysis. Semin Arthritis Rheum. 2018;47(5):689-702.
79 Zhao Y, Xu L, Tian D, Xia P, Zheng H, Wang $\mathrm{L}$, et al. Effects of sodium-glucose co-transporter 2 (SGLT2) inhibitors on serum uric acid level: a meta-analysis of randomized controlled trials. Diabetes Obes Metab. 2018; 20(2):458-62.

80 Juraschek SP, Appel LJ, Miller ER 3rd. Metoprolol increases uric acid and risk of gout in African Americans with chronic kidney disease attributed to hypertension. Am J Hypertens. 2017;30(9):871-5.

81 Musini VM, Nazer M, Bassett K, Wright JM. Blood pressure-lowering efficacy of monotherapy with thiazide diuretics for primary hypertension. Cochrane Database Syst Rev. 2014;5(5):CD003824.
82 Schlesinger N. The safety of treatment options available for gout. Expert Opin Drug Saf. 2017;16(4):429-36.

83 Bove M, Cicero AFG, Borghi C. The effect of xanthine oxidase inhibitors on blood pressure and renal function. Curr Hypertens Rep. 2017;19(12):95.

84 Stamp LK, Merriman TR, Singh JA. Expert opinion on emerging urate-lowering therapies. Expert Opin Emerg Drugs. 2018;23(3): 201-9. 